\title{
A Novel, Extremely Bioavailable Cocrystal of Pterostilbene
}

Lídia Bofill, Rafael Barbas, Dafne de Sande, Mercè Font-Bardia, Clara Ràfols, Joan Albertí, and Rafel Prohens*

a Departament de Química, Universitat Autònoma de Barcelona, 08913 Bellaterra, Barcelona, Spain.

${ }^{\mathrm{b}}$ Departament de Mineralogia, Petrologia i Geologia Aplicada, Universitat de Barcelona, Martí i Franquès s/n, 08028 Barcelona, Spain.

${ }^{c}$ Unitat de Difracció de Raigs-X, Centres Científics i Tecnològics de la Universitat de Barcelona (CCiTUB), Universitat de Barcelona, Solé i Sabarís, 1-3, 08028 Barcelona, Spain.

\section{* Corresponding Author}

Rafel Prohens - Center for Intelligent Research in Crystal Engineering S.L., Parc Científic de Barcelona, E-08028 Barcelona, Spain; Unitat de Polimorfisme i Calorimetria, Centres Científics i Tecnolo `gics, Universitat de Barcelona, E-08028 Barcelona, Spain; orcid.org/0000-0003-0294-1720; Email: rafel@ccit.ub.edu 


\begin{abstract}
New multicomponent solid forms of the nutraceutical pterostilbene have been discovered and characterized through experimental cocrystal screening. Among the coformers tested, picolinic acid formed a cocrystal with a 10 -fold enhancement of oral bioavailability in rats, which converts the new cocrystal into a very promising candidate for new formulations of pterostilbene with improved performance.
\end{abstract}

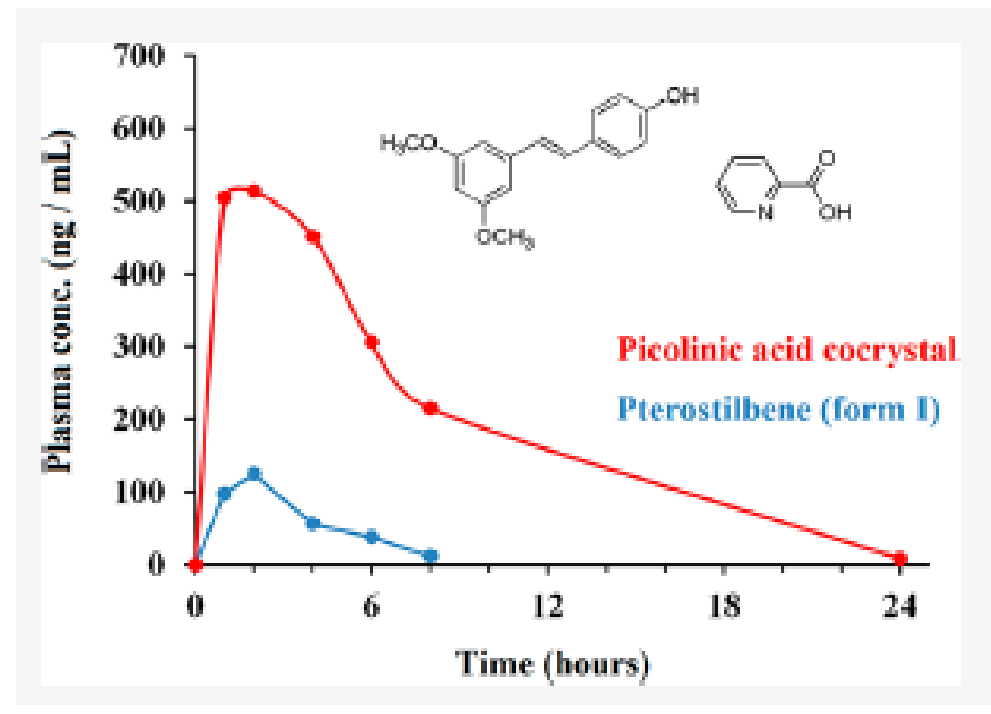




\section{Introduction}

Nutraceutical ingredients are natural compounds, usually extracted and purified from both food and nonfood plants. 1 In recent years, nutraceuticals have been experienced an increased interest because their presumed safety and potential therapeutic effects allow health claims as food supplements with a variety of health benefits for humans and animals. 2

Pterostilbene (trans-3,5-dimethoxy-4'-hydroxystilbene) is a phytoalexin found in fruits such as grapes, deerberries, blueberries, and peanuts and in wine.3,4 It has been evidenced that pterostilbene may have preventive and therapeutic properties in a long list of human diseases which include neurological,5,6 cardiovascular,7,8 metabolic, 9,10 and hematologic disorders.11,12 In addition, it has been reported that it can act as a nontoxic modulator of anticancer response in drugs such as sertraline and gefitinib.13

Pterostilbene is known to exist as a variety of polymorphs, and the crystal structures of forms I-IV have been reported recently by some of us.14,15 It is structurally similar to resveratrol, another nutraceutical present in red wine but with a better human bioavailability probably due to its higher lipophilicity, 16 which has motivated their use as dietary supplements claiming antioxidant,17 anti-inflammatory, 18 and anticarcinogenic properties.19 However, in the search for a safe formulation of a nutraceutical it is desirable to have a further increase in bioavailability because this allows reduction of as much as possible the side effects by reducing the dosage. For instance, it has been recently reported that pterostilbene could cause an increment of low-density lipoprotein (LDL) cholesterol20 in humans. Thus, a more bioavailable formulation of pterostilbene could preserve the health benefits with a reduced effect of known or unknown side effects.

In this sense, the use of crystal engineering approaches has produced successful and promising case studies of both nutraceutical and pharmaceutical compounds with bioavailability enhancement.21,22 This is because each solid form of a drug compound shows unique properties and some of them can offer certain advantages with regard to solubility and dissolution rate, which converts cocrystal formation into a viable strategy toward improving the pharmacokinetic data of active ingredients. Thus, the discovery of multicomponent solid forms of nutraceuticals is currently a very active field of research which requires the selection of acceptable coformers in order to form cocrystals with the nutraceutical having suitable physicochemical properties. 23

Moreover, the importance of the coformer is not just structural (i.e., a compound able to reduce the free energy of the system by establishing strong intermolecular interactions and favoring efficient packing) since its role as a counter partner can provide to the new entity not only with advantageous physicochemical properties but also with the ability to generate potential synergetic health benefits in a single solid form that can also be exploited.24

On the other hand, due to the low solubility of pterostilbene, the development of potential new oral formulations has been focused on increasing the aqueous solubility of this compound by using hydroxypropyl- $\beta$-cyclodextrin as a complexing agent 25 or by cocrystal formation 26 using as coformers piperazine and caffeine 27 and carbamazepine and glutaric acid.28 Actually, the commercial pterostilbene:caffeine cocrystal PURENERGY has been commercially available in the US market for years (Chromadex, USA).

In this manuscript we report the discovery and characterization of eight pterostilbene cocrystals by following a multicomponent solid form experimental screen. Among the new forms, a picolinic acid cocrystal showed a remarkable enhancement in both dissolution profile and bioavailability, which makes it a very promising candidate for more efficient formulations at a lower dosage of this important nutraceutical compound. 


\section{EXPERIMENTAL SECTION}

2.1. Materials. Pterostilbene was purchased from Dynveo. Details of the purification and characterization of pterostilbene (form II) can be found in the Supporting Information. The coformers picolinic acid, ethylenediamine, 1,4-diazabicyclo[2.2.2]octane (DABCO), 1,4,8,11tetraazacyclotetradecane, 1,4-dimethylpiperazine, theophylline, and 2,3,5-trimethylpyrazine were purchased from Sigma-Aldrich.

2.2. Methods. 2.2.1. Coformer Selection. A list of 58 coformers with potentially acceptable toxicity were tested (see section 1.1 in the Supporting Information for further details). We recently reported 24 the molecular electrostatic potential surface (MEPS) computed at the DFT level of computation, and it revealed that the pterostilbene molecule has highly negative values of MEPS located at the $\mathrm{O}$ atoms of the methoxy and phenol groups and a highly positive value at the phenolic $\mathrm{H}$ atom, as expected (Figure 1). Thus, when its dual electrostatic nature is taken into account, coformers having both strong hydrogen bond donor and acceptor groups such as carboxylic acids, hydroxybenzoic acids, phenols, and amine compounds, having the potential to interact satisfactorily with the methoxy and phenol functional groups of pterostilbene, were chosen.

2.2.2. Solubility Determination. In order to choose the most suitable solvents in which the screening would be performed, a qualitative determination of solubility in 30 solvents was conducted for both pterostilbene (form II) and each coformer. Pterostilbene (20mg, 0.078 $\mathrm{mmol}$ ) was suspended in each solvent, using a maximum volume of $2.0 \mathrm{~mL}$ at room temperature. When the sample was not soluble, the temperature was increased to a maximum of $90{ }^{\circ} \mathrm{C}$. In this qualitative solubility determination, solvents are classified as soluble, insoluble, or partially soluble at each temperature (see section 1.2 in the Supporting Information). The same procedure has been followed with all of the coformers, and for this study mainly solvents in which both components (pterostilbene and coformer) were partially soluble at $25{ }^{\circ} \mathrm{C}$ were selected to be tested in the grinding experiments.

2.2.3. Cocrystal Screening Methodologies. 2.2.3.1. Grinding Methodologies. Screening through net grinding (with liquid coformers) and liquid-assisted grinding experiments (LAG) was conducted by grinding 20-50 mg of a 1:1 mixture of pterostilbene and each coformer together with one drop of different solvents using a Retsch MM 2000 grinding mill. The samples were placed in $2 \mathrm{ml}$ volume stainless steel jars, along with two stainless tungsten grinding balls of $3 \mathrm{~mm}$ diameter. Grinding was performed for 15-30 min, with a mill frequency of $30 \mathrm{~Hz}$. Finally, the samples were collected immediately without prior drying for PXRD analysis. The formation of a new solid form was determined by comparing PXRD patterns of the starting materials (any known crystal form of both pterostilbene and each coformer) and the LAG experiment products.

2.2.3.2. Reaction Crystallization Methodology. When evidence of new forms was detected, reaction crystallization experiments (RC) were conducted by preparing a saturated solution of the most soluble component (pterostilbene or coformer) in a suitable solvent in a sealed vial under stirring. A small quantity of the less soluble component was added until it did no longer dissolve. The suspension was stirred at different times, and the resulting solids were filtered and analyzed by PXRD.

2.2.3.3. Solvent-Mediated Transformation Methodology. Screening through solvent-mediated transformations (SMT) was conducted by preparing suspensions of pterostilbene and the coformer in different molar ratios (40-300 $\mathrm{mg}$ of the final mixture) in suitable solvents. The sealed vials were stirred for different times, and the resulting solids were filtered and analyzed by PXRD. 
2.2.3.4. Solution Crystallization Methodologies. Solutions of pterostilbene and coformer mixtures in different molar ratios (20-1100 $\mathrm{mg}$ of the final mixture) were prepared in different solvents at $25^{\circ} \mathrm{C}$ and heated (to 50 or $60^{\circ} \mathrm{C}$ ) in a stainless steel heating block. The heater was switched off, and the solutions were slowly cooled to $25^{\circ} \mathrm{C}$ inside the heating block. The samples that did not crystallize were tightly sealed and kept at $4-8$ or $25{ }^{\circ} \mathrm{C}$ until precipitation was observed.

2.2.3.5. Preparation of the New Solid Forms: Scale-up Batches. Experimental procedures for the preparation of picolinic acid and ethylenediamine cocrystals have been optimized, and several scale up batches were prepared for dissolution rate and pharmacokinetics studies. A summary of the experimental procedures can be found in Table S1 in the Supporting Information.

2.2.4. X-ray Crystallographic Analysis. Single-crystal X-ray diffraction (SCXRD) intensity data of the different crystal forms of pterostilbene were collected using a D8 Venture system equipped with a multilayer monochromator and a Mo microfocus $(\lambda=0.71073 \AA)$. Frames were integrated with the Bruker SAINT software package using a SAINT algorithm. Data were corrected for absorption effects using the multiscan method (SADABS).30 The structure was solved and refined using the Bruker SHELXTL software package, a computer program for the automatic solution of crystal structures, and refined by a full-matrix least-squares method with ShelXIe Version 4.8.0, a Qt graphical user interface for the SHELXL computer program.31

Powder X-ray diffraction (PXRD) patterns were obtained on a PANalytical X'Pert PRO MPD diffractometer in transmission configuration using $\mathrm{Cu} K \alpha 1+2$ radiation $(\lambda=1.5406 \AA$ ) with a focusing elliptical mirror and a PIXcel detector working at a maximum detector active length of $3.347^{\circ}$. Configuration of the convergent beam with a focalizing mirror and a transmission geometry with a flat sample sandwiched between low-absorbing films measuring from 2 to $40^{\circ}$ in $2 \theta$, with a step size of $0.026^{\circ}$ and a total measurement time of 8-30 min at room temperature $(298 \mathrm{~K})$. The powder diffractogram of the picolinic acid cocrystal was indexed, the lattice parameters were refined by means of LeBail fits using Dicvol04,32 and the space group was determined from the systematic absences. A summary of crystal data and relevant refinement parameters is given in Table 1.

2.2.5. Differential Scanning Calorimetry (DSC). Differential scanning calorimetry analysis was carried out by means of a Mettler-Toledo DSC-822e calorimeter. Experimental conditions: aluminum crucibles of $40 \mu \mathrm{L}$ volume, atmosphere of dry nitrogen with $50 \mathrm{~mL} / \mathrm{min}$ flow rate, heating rate of $10^{\circ} \mathrm{C} / \mathrm{min}$. The calorimeter was calibrated with indium of $99.99 \%$ purity (mp $156.5^{\circ} \mathrm{C}, \Delta \mathrm{H}=28.42 \mathrm{~J} / \mathrm{g}$ ).

2.2.6. Thermogravimetric Analysis (TGA). Thermogravimetric analysis was performed on a Mettler-Toledo TGA-851e thermobalance. Experimental conditions: alumina crucibles of $70 \mu \mathrm{L}$ volume, atmosphere of dry nitrogen with $50 \mathrm{~mL} / \mathrm{min}$ flow rate, heating rate of $10^{\circ} \mathrm{C} / \mathrm{min}$.

2.2.7. Nuclear Magnetic Resonance (NMR). Proton nuclear magnetic resonance (1H NMR) spectra were recorded on a Varian Mercury 400 instrument $(400 \mathrm{MHz})$. Chemical shifts for protons are reported in parts per million (ppm) downfield from tetramethylsilane and are referenced to residual protons in the NMR solvents (dmso-d6, $\delta$ 2.50; acetone-d6, $\delta$ 2.05; chloroform-d, $\delta$ 7.26). Experimental conditions, delay, $1 \mathrm{~s}$; pulse, $45^{\circ}$; scans, 32.

2.2.8. Dissolution Rate Determination. The small-scale GI dissolution method33 was followed for dissolution rate measurements. First, tablets of $3 \mathrm{~mm}$ diameter were made using a manual hydraulic tablet press (Applied Measurements Ltd., U.K.). The applied pressure was $100 \mathrm{~kg}$ for $2 \mathrm{~min}$. A 10-15 mg portion of the compound, pterostilbene or cocrystals, was weighed. The total exposed surface area was $0.5 \mathrm{~cm} 2$.

Dissolution tests were performed in FaSSIF v2 medium (pH 6.5 using phosphoric acid $28.4 \mathrm{mM}$ ) with a GlpKa titrator (Sirius Analytical Instruments, U.K.). Dissolution data were 
recorded for $120 \mathrm{~min}$ at $25^{\circ} \mathrm{C}$. The procedure was as follows: $15 \mathrm{~mL}$ of FaSSIF v2 medium was placed in the sample vial containing the tablet. Spectral collection started immediately after. Spectra were collected every $30 \mathrm{~s}$ between 250 and $450 \mathrm{~nm}$ through a Sirius D-PAS spectrometer with a bifurcated optic fiber dip probe (Hellma Analytics). The medium was stirred at a constant rate. The spectroscopic data were converted to the concentration of pterostilbene by applying the Beer-Lambert law using the molar extinction coefficient of pterostilbene determined in FaSSIF V2 by UV-metric titration. Spectral regions where the signal was saturated $(A>1.5)$ or presented medium interferences were discarded. Then, the concentration data were converted into absolute sample quantities and used to generate the graphs showing the sample quantity vs time.

The dissolution rate was obtained through the fit of the first-order Noyes-Whitney exponential equation to the data: $[X] t=S(1-e-k d(t-t 0))$. In this equation, $[X] t$ is the weight in grams of compound in solution at the experiment time $(\mathrm{min}), \mathrm{S}$ is its extrapolated solubility (g), $\mathrm{kd}$ is the rate constant for dissolution ( $\mathrm{min}-1)$, and to ( $\mathrm{min}$ ) is a term allowing for a temporal offset. $\mathrm{S}, \mathrm{kd}$, and to are refined in order to minimize the root-mean-square deviation between the modeled concentrations and the measured concentrations. The dissolution rate $(\mathrm{g} \min -1)$ is given by the product kdS.

2.2.9. Rat Pharmacokinetics. Pharmacokinetic study in rats was conducted the Unitat de Toxicologia Experimental i Ecotoxicologia (UTOX-PCB, Parc Cientifí c de Barcelona, Spain). This pharmacokinetic study was carried in compliance with the current guidelines and animal handling protocols reviewed and approved by the Institutional Animal Care Committee of the Parc Cientifí c of Barcelona.

Ten male Sprague-Dawley rats ( $420 \mathrm{~g})$ were used in the study and kept in overnight fasting state before administrations. Formulations of pterostilbene $(20 \mathrm{mg} / \mathrm{kg}$, free basis) and a pterostilbene:picolinic acid cocrystal $(20 \mathrm{mg} / \mathrm{kg}, 13.5 \mathrm{mg} / \mathrm{kg}$ pterostilbene) were prepared as aqueous suspensions in $0.5 \%$ carboxymethylcelullose (CMC). Both formulations were administered by oral gavage $(10 \mathrm{~mL} / \mathrm{kg}$ body weight). Blood samples $(\sim 0.3 \mathrm{~mL})$ taken from the tail vein were collected in EDTA.K3 tubes at predose and 1, 2, 4, 6, 8, and $24 \mathrm{~h}$ after administration and centrifuged at $1500 \mathrm{~g}$ for $10 \mathrm{~min}$ to obtain plasma. Plasma samples were stored at $-70^{\circ} \mathrm{C}$ until UHPLC-MS/MS analysis.

2.2.10. Plasma Bioanalysis. Concentrations of pterostilbene in rat plasma samples were determined at the Department of Chemical Engineering and Analytical Chemistry (University of Barcelona, Spain) by ultrahigh-pressure liquid chromatography coupled to tandem mass spectrometry (UHPLC-MS/MS).

Plasma samples $(100 \mu \mathrm{L})$ were processed using protein precipitation with acetonitrile $(1000 \mu \mathrm{L})$. After vortex treatment(30 s) and centrifugation (4800 rpm for $15 \mathrm{~min}$ ), supernatants were evaporated to dryness under an N2 stream and reconstituted in $200 \mu \mathrm{L}$ of methanol/0.025\% ammonia $(3 / 1, \mathrm{v} / \mathrm{v})$ and transferred to an autosampler vial. The UHPLCMS/MS measurements were carried out on an Open Accela UHPLC instrument (Thermo Fisher Scientific, San Jose, CA, USA), equipped with a quaternary pump and a CTC autosampler. The UHPLC instrument was coupled to a TSQ Quantum Ultra AM triple-quadrupole mass analyzer (Thermo Fisher Scientific), equipped with hyperbolic quadrupoles and a heated-electrospray ionization (H-ESI) source. Chromatography consisted of a Kinetex C18 column (100 mm $\times 4.6$ $\mathrm{mm}, 2.6 \mu \mathrm{m}$ ) from Phenomenex (Torrance, CA, USA) at room temperature with a gradient elution mode using $0.025 \%$ ammonia aqueous solution (solvent $A$ ) and methanol (solvent $B$ ) at a flow rate of $0.5 \mathrm{~mL} / \mathrm{min}$.monitoring in negative ESI (electrospray ionization) mode (m/z $255 \rightarrow$ 240). The lower limit of quantitation was $5 \mathrm{ng} / \mathrm{mL}$, and the linear calibration range was 5-1000 $\mathrm{ng} / \mathrm{mL}$.

2.2.11. Pharmacokinetic Analysis. Pharmacokinetic analysis was performed by noncompartmental methods using the Kinetica version 5 software (Alfasoft Limited, Luton, U.K.). 


\subsection{Synthesis and Characterization of the New Cocrystals.}

We have performed a comprehensive cocrystal screen with a set of coformers, which includes a broad array of compounds (see section 1.1 in the Supporting Information) from a variety of 28 organic solvents, which produced 634 individual crystalline solids (see Table S1 in the Supporting Information). Evidences of new solid phases with 37 out of all the coformers tested (see Table S1 in the Supporting Information) with net grinding or LAG experiments were detected. On the other hand, cocrystal formation with 7 out of all the coformers tested, picolinic acid, ethylenediamine, 1,4-diazabicyclo[2.2.2] octane (DABCO), 1,4,8,11-tetraazacyclotetradecane, 1,4-dimethylpiperazine, 2,3,5-trimethylpyrazine, and theophylline, was confirmed through reaction crystallization and solventmediated transformation experiments. As a result of the cocrystal screen 8 new multicomponent solid forms were discovered with 7 out of the 58 coformers tested.

Synthesis details of bulk powder and single crystals together with characterization of pterostilbene cocrystals can be found in sections 2-4 in the Supporting Information. The stoichiometry was assessed on the basis of NMR data when the crystal structure was not available. TGA was used to confirm or discard the presence of water/solvent in the structure. Qualitative comparisons of PXRD diffractograms of bulk cocrystals and those simulated from the cif files were conducted in order to confirm the identity of the crystal form, and they are shown in the Supporting Information. Differences are due to the thermal dilatation, since the crystal structures were solved at $100 \mathrm{~K}$ while the PXRD diagrams were obtained at $298 \mathrm{~K}$.

2.3.1. Pterostilbene:Picolinic Acid Cocrystal (1:1). The bulk powder was obtained by reaction crystallization in toluene at $25^{\circ} \mathrm{C}$. The $1: 1$ stoichiometry was compatible with the integration of the most relevant $1 \mathrm{H}$ NMR signals in chloroform and with the volume calculated from PXRD indexed cell parameters $(a=15.920$ (1) $\AA, b=9.5200(8) \AA, c=12.870(1) \AA$, $\left.\beta=100.345(7)^{\circ}, V=1918.7(3) \AA ̊ 3, \operatorname{Rwp}=6.68 \%\right)$.

2.3.2. Pterostilbene:Ethylenediamine Cocrystal (2:1). The bulk powder was obtained by slow evaporation of a solution of pterostilbene in ethylenediamine at $25{ }^{\circ} \mathrm{C}$. According to its crystal structure it can be attributed to a new form containing a half-molecule of ethylenediamine per one molecule of pterostilbene.

2.3.3. Pterostilbene:Ethylenediamine:Water Cocrystal Hydrate $(2: 1: 2)$. The bulk powder was obtained by slurrying pterostilbene in ethylenediamine during a scale-up process at $25{ }^{\circ} \mathrm{C}$. The 2:1:2 stoichiometry was compatible with the integration of the most relevant $1 \mathrm{H}$ NMR signals in chloroform and with the volume calculated from PXRD indexed cell parameters $\left(a=26.745(3) \AA, b=5.1961(4) \AA, c=22.971(2) \AA, \beta=100.526(6)^{\circ}, V=3138.5(6) \AA 3\right.$, Rwp $=7.99$ ). The amount of water was determined by TGA.

2.3.4. Pterostilbene:DABCO Cocrystal (2:1). The bulk powder was obtained by reaction crystallization in xylene at $25^{\circ} \mathrm{C}$. According to its crystal structure it can be attributed to a new form containing one molecule of DABCO per two molecules of pterostilbene.

2.3.5. Pterostilbene:1,4,8,11-Tetraazacyclotetradecane Cocrystal (2:1). The bulk powder was been obtained by slow evaporation in chloroform at $25^{\circ} \mathrm{C}$. According to its crystal structure it can attributed to a new form containing a half-molecule of 1,4,8,11tetraazacyclotetradecane per one molecule of pterostilbene.

2.3.6. Pterostilbene:1,4-Dimethylpiperazine Cocrystal (2:1). The bulk powder was obtained by slurrying pterostilbene in 1,4-dimethylpiperazine at $25^{\circ} \mathrm{C}$. According to its crystal structure it can be attributed to a new form containing a half-molecule of 1,4dimethylpiperazine per one molecule of pterostilbene.

2.3.7. Pterostilbene:Theophylline:DCM Cocrystal Solvate (1:1:1). The bulk powder was obtained by reaction crystallization in dichloromethane at $25^{\circ} \mathrm{C}$. According to its crystal structure it can be attributed to a new form containing one molecule of theophylline and 
one molecule of dichloromethane per 1 molecule of pterostilbene. This cocrystal is the subject of a computational study we have reported elsewhere.24

2.3.8. Pterostilbene:2,3,5-Trimethylpyrazine cocrystal (2:1). The bulk powder was obtained by slurrying pterostilbene in 2,3,5-trimethylpyrazine at $25^{\circ} \mathrm{C}$. According to its crystal structure it can be attributed to a new form containing a half-molecule of 2,3,5trimethylpyrazine per one molecule of pterostilbene. 


\section{RESULTS AND DISCUSSION}

3.1. Crystal Structure Analysis. The crystal structures of the ethylenediamine, DABCO, 1,4,8,11-tetraazacyclotetradecane, 1,4-dimethylpiperazine, and 2,3,5trimethylpyrazine cocrystals were solved by single crystal X-ray diffraction. Single crystals of the picolinic acid cocrystal of suitable quality could not be obtained; thus, we indexed a good-quality powder diffractogram obtained from a pure polycrystalline material and refined the lattice parameters by means of LeBail fits. A 1:1 stoichiometry was deduced from $1 \mathrm{H}$ NMR experiments and the indexed cell volume.

3.1.1. Crystal Structure of the Pterostilbene:Ethylenediamine Cocrystal. The crystal structure has one molecule of pterostilbene and a half-molecule of ethylenediamine in the asymmetric unit (Figure 2). As expected, a strong hydrogen bond is established between the pterostilbene phenol group and the primary amine. Moreover, $\mathrm{CH} \cdots \pi$ interactions established between the methoxy hydrogens and the dimethoxy aromatic ring ( $\mathrm{dH}$-centroid $=$ $2.71 \AA$ ) and between methoxy hydrogens and benzene hydrogens with phenol aromatic ring ( $\mathrm{dH}$-centroid $=2.89$ and $2.96 \AA$, respectively), together with methoxy-methoxy interactions forming an R2 2(14) supramolecular synthon, are the most relevant secondary forces in the crystal.

3.1.2. Crystal Structure of the Pterostilbene:DABCO Cocrystal. The crystal structure has two molecules of pterostilbene and one molecule of DABCO in the asymmetric unit (Figure 3). Again, a strong hydrogen bond is established between the pterostilbene phenol group and the primary amine, together with $\mathrm{CH} \cdots \pi$ interactions established between the methoxy hydrogens and the dimethoxy aromatic ring (dH-centroid $2.91 \AA$ ). Moreover, a R2 2(6) supramolecular synthon is established between methoxy groups. Static disorder is present in the DABCO molecule, and two different positions have been determined with different occupancy factors ( $52.4 \%$ and $46.7 \%$, respectively).

3.1.3. Crystal Structure of the Pterostilbene:1,4,8,11Tetraazacyclotetradecane Cocrystal. The crystal structure has one molecule of pterostilbene and a half-molecule of 1,4,8,11-tetraazacyclotetradecane in the asymmetric unit (Figure 4). Each tetraamine molecule establishes strong hydrogen bonds with pterostilbene phenol groups by using two of the four nitrogen atoms, forming 2:1 sandwich-type assemblies, which are packed in a linear fashion.

3.1.4. Crystal Structure of Pterostilbene:1,4-Dimethylpiperazine Cocrystal. The crystal structure has one molecule of pterostilbene and a half-molecule of 1,4dimethylpiperazine in the asymmetric unit (Figure 5). Both nitrogen atoms of the piperazine ring establish strong hydrogen bonds with pterostilbene phenol groups, and the $\mathrm{CH} \cdots \pi$ interactions are in this case established between the methoxy hydrogens and the phenol aromatic ring (dH-centroid $2.73 \AA$ ). Again, a R2 2(6) supramolecular synthon is formed between methoxy groups. Interestingly, methoxy groups are in the less stable configuration, which is in a counterclockwise direction with the methoxy groups pointing to opposite directions.

3.1.5. Crystal Structure of the Pterostilbene:2,3,5-Trimethylpyrazine

Cocrystal. The crystal structure has two molecules of pterostilbene (one having the methoxy groups in a clockwise and the other in a counterclockwise configuration) and one molecule of 2,3,5-trimethylpyrazine in the asymmetric unit (Figure 6). Similarly to previous structures, both nitrogen atoms of the pyrazine ring establish strong hydrogen bonds with pterostilbene phenol groups. Remarkably, an uncommon $\pi(\mathrm{C}$ ? $\mathrm{C}) \cdots$ pyrazine ring interaction (distances between the pyrazine centroid and the carbon atoms of the double bond are 3.37 and $3.41 \AA$ ) is established between the surface of the pterostilbene double bond, which is $\pi$-basic (electron rich), and that of the pyrazine ring, which is particularly $\pi$-acidic (electron poor) due to the strong hydrogen 
bonds formed with the phenol groups. Actually, a similar synergistic effect has been computationally studied in pyrazine complexes, where anion- $\pi$ interactions are reinforced as a consequence of halogen bonds established by the nitrogen atoms.34 Moreover, $\pi \cdots \pi$ interactions are established between the dimethoxy aromatic ring and the pyrazine ring (dcentroid-centroid $3.55 \AA$ A). Finally, $\mathrm{CH} \cdots \pi$ interactions are established between the methoxy hydrogens and the phenol aromatic ring ( $\mathrm{dH}$-centroid $=2.73$ and $2.76 \AA$, respectively).

3.2. Dissolution Profiles. In order to gather some basic information about the effect of the coformer on the dissolution rate, two of the new cocrystals having GRAS status and different chemical functionalities were chosen for a preliminary dissolution profile determination. Ethylenediamine and picolinic acid cocrystals were tested in FaSSIF v2 medium together with pterostilbene form I and the commercial caffeine cocrystal for comparative purposes. Figure 7 shows their average dissolution rate curves. While in tablets of pure pterostilbene the amount of pterostilbene in solution is still increasing after 120 min of dissolution, the cocrystals dissolve at a higher rate, reaching a concentration plateau after 20 and $60 \mathrm{~min}$ for picolinic acid and ethylenediamine cocrystals, respectively. The amount of pterostilbene in solution achieved with the dissolution of the cocrystals is 3-4 times higher than the amount reached after $2 \mathrm{~h}$ of dissolution of the pure compound. Although the ethylenediamine cocrystal takes longer to reach the final pterostilbene concentration, the solution obtained through the dissolution of this cocrystal is the one that contains a greater amount of dissolved pterostilbene.

Table 2 shows the dissolution rate of the studied solid forms measured in the UV range of $300-400 \mathrm{~nm}$ (for pterostilbene) and $350-400 \mathrm{~nm}$ (for the cocrystals), obtained in a $50 \mathrm{~min}$ time frame. As expected, these exploratory data confirmed that very water-soluble coformers increase greatly the solubility of pterostilbene cocrystals and suggested that the bioavailability could also be favorably affected. 27

3.3. Pharmacokinetics Study. We decided to focus on the picolinic acid cocrystal (rather than the amine cocrystals) because the promising dissolution profile together with its GRAS status made it a good candidate for a potential formulation. Picolinic acid is an endogenous metabolite of Ltryptophan (TRP), which has been reported to possess health benefits such as neuroprotective, immunological, and antiproliferative properties. 35 It is inexpensive, safe, and the most important human chelator for metal ions, such as Zn2+ and Fe2+. Moreover, chromium picolinate is used as a dietary supplement that may promote weight loss.36 The mean plasma pterostilbene concentration:time profiles after oral administration of commercial pterostilbene $(20 \mathrm{mg} / \mathrm{kg})$ and pterostilbene:picolinic acid cocrystal $(20 \mathrm{mg} / \mathrm{kg}, 13.5$ $\mathrm{mg}$ as pterostilbene) are represented in Figure 8. The pharmacokinetic parameters of pterostilbene after oral administration of commercial pterostilbene $(20 \mathrm{mg} / \mathrm{kg})$ and pterostilbene:picolinic acid cocrystal $(20 \mathrm{mg} / \mathrm{kg}, 13.5 \mathrm{mg}$ as pterostilbene) are summarized in Table 3. The systemic exposure to pterostilbene was 4.5-fold and 6.7-fold greater in Cmax and AUCinf, in spite of the fact that the cocrystal dose was not equimolar with that of commercial pterostilbene. The variability in the tmax value was higher for the cocrystal formulation and ranked from 1 to $6 \mathrm{~h}$, which translated into a greater variability in the Cmax values found in comparison to the commercial pterostilbene. The relative oral bioavailability (pterostilbene dose corrected) was 9.9-fold higher following administration as a cocrystal, which was in agreement with the substantial increase in the solubility rate observed for this cocrystal in the dissolution test conducted in FaSSIF medium. These results demonstrate that the aqueous solubility of pterostilbene plays a key role in its oral absorption.In comparison, the oral administration of a commercial pterostilbene:caffeine cocrystal (1:1)28 (PURENERGY, Chromadex, USA) provided a modest increase of 1.5 -fold on the oral availability of pterostilbene versus a commercial pterostilbene.37 Therefore, the pterostilbene:picolinic acid cocrystal (1:1) developed in this study is an excellent solid oral formulation with an excellent potential to maximize the oral absorption of pterostilbene. 
Following intravenous administration of different doses of pterostilbene $(2.5,10$, and 25 $\mathrm{mg} / \mathrm{kg}$ ) to rats, this compound showed dose-dependent pharmacokinetics. This lack of dose proportionality was the cause for around a 2-fold reduction in the systemic clearance at higher doses. This clearance reduction can be explained by a saturation of the major phase II elimination pathway of pterostilbene in rats, leading to a limited elimination of pterostilbene at higher doses. 38

The oral bioavailability in the fasted state of different formulations of pterostilbene has been widely studied in the literature.25,37-39 Following oral administration of pterostilbene, the estimation of the absolute oral bioavailability was highly variable and dependent on the formulation used, the oral doses administered, and the pharmacokinetic intravenous data used in calculations. This is consistent with the dosedependent proportionality showed by pterostilbene after oral and intravenous administration. The absolute oral bioavailability ranged from $59 \%(15 \mathrm{mg} / \mathrm{kg})$ to $116 \%(60 \mathrm{mg} / \mathrm{kg})$ on administration as a hydroxypropyl- $\beta$-cyclodextrin formulation 38 and from $67 \%(56 \mathrm{mg} / \mathrm{kg})$ and $94 \%(168 \mathrm{mg} / \mathrm{kg})$ on administration in $0.5 \%$ methylcellulose- $0.2 \%$ Tween 80 suspensions. 39 This high variability observed between studies must be evaluated in the context of the lack of dose proportionality showed by pterostilbene after administration of high oral doses. Therefore, for a proper estimation of the absolute oral bioavailability of pterostilbene, one needs to take into account the systemic exposure (AUCinf) obtained by an intravenous route as a reference value, which should be on the same order as that found after oral administration. In the two previous publications,37,39 the AUCinf value after the intravenous route used in calculations was substantially lower than that obtained after administration of oral doses greater than $10 \mathrm{mg} / \mathrm{kg}$; therefore, the absolute oral bioavailabilities of these formulations at those doses were clearly overestimated.

Although the intravenous pharmacokinetics in rats of pterostilbene was not evaluated in this study either as such or in the cocrystal, we can estimate the absolute oral bioavailability of the pterostilbene:picolinic acid cocrystal, taking as a reference the systemic exposure observed (AUCinf) described in the literature.38,39 After an intravenous administration of 10 $\mathrm{mg} / \mathrm{kg}$,38 the observed AUCinf value $(2812 \mathrm{ng} \mathrm{h} / \mathrm{mL}$ ) was comparable to that found after oral administration (13.5 mg/kg as pterostilbene) of our cocrystal (AUCinf $4699 \mathrm{ng} \mathrm{h} / \mathrm{mL}$ ), which provides an absolute oral bioavailability of $125 \%$. When the same exercise is conducted with the intravenous PK data ( $11.2 \mathrm{mg} / \mathrm{kg}$ ) obtained by Kapetanovic et al.39 with an AUCinf value of 4090 $\mathrm{ng} \mathrm{h} / \mathrm{mL}$, the estimated absolute oral bioavailability of pterostilbene with our cocrystal would be $97 \%$. These results confirm that the oral bioavailability of pterostilbene in rats following administration of the pterostilbene:picolinic acid cocrystal (1:1) is excellent and is close to $100 \%$, which makes it the best potential solid oral formulation of pterostilbene. 


\section{CONCLUDING REMARKS}

Nutraceutical compounds offer a long list of potential health benefits for humans, and in particular pterostilbene has been involved in important scientific studies intended to explore its application in different areas of health. However, the low bioavailability of many nutraceutical compounds have hindered an efficient application of their potential health benefits in humans. Our work demonstrates that crystal engineering can be very suitable to approach this intrinsic problem associated with lipophilic compounds in the form of a cocrystal. Following oral administration to rats, the pterostilbene:picolinic acid cocrystal (1:1) increased by 9.9 -fold the relative oral bioavailability of pterostilbene in comparison with its commercial oral solid form. These results confirm that the oral disposition of this cocrystal is excellent and the cocrystal has a great potential to be one of the best oral solid formulations for pterostilbene in humans.

\section{Notes}

The authors declare no competing financial interest.

\section{Acknowledgements}

We thank Centre for the Development of Industrial Technology (CDTI) for financial support (Neotec SNEO-20161021/CRYSTACURE project). 


\section{References}

(1) Shi, J. Functional Food Ingredients and Nutraceuticals Processing Technologies, 2nd ed.; CRC Press: 2015.

(2) Bagchi, D.; Preuss, H. G.; Swaroop, A. Nutraceuticals and Functional Foods in Human Health and Disease Prevention, 1st ed.; CRC Press: 2015.

(3) Roupe, K. A.; Remsberg, C. M.; Yáñez, J. A.; Davies, N. M. Pharmacometrics of stilbenes: seguing towards the clinic. Curr. Clin. Pharmacol. 2006, 1, 81-101.

(4) Ruiz, M. J.; Fernández, M.; Picó, Y.; Mañes, J.; Asensi, M.; Carda, C.; Asensio, G.; Estrela, J. M. Dietary Administration of High Doses of Pterostilbene and Quercetin to Mice Is Not Toxic. J. Agric. Food Chem. 2009, 57, 3180-3186.

(5) Poulose, S. M.; Thanthaeng, N.; Miller, M. G.; Shukitt-Hale, B. Effects of pterostilbene and resveratrol on brain and behaviour. Neurochem. Int. 2015, 89, 227-233.

(6) Chang, J.; Rimando, A.; Pallas, M.; Camins, A.; Porquet, D.; Reeves, J.; Shukitt-Hale, B.; Smith, M. A.; Joseph, J. A.; Casadesus, G. Low-dose pterostilbene, but not resveratrol, is a potent neuromodulator in aging and Alzheimer's disease. Neurobiol. Aging 2012, 33, 2062-2071.

(7) Park, E.; Lim, Y.; Hong, J.; Yoo, H.; Lee, C.; Pyo, M.; Yun, Y. Pterostilbene, a natural dimethylated analog of resveratrol, inhibits rat aortic vascular smooth muscle cell proliferation by blocking Aktdependent pathway. Vasc. Pharmacol. 2010, 53, 61-67.

(8) Zhang, L.; Zhou, G.; Song, W.; Tan, X.; Guo, Y.; Zhou, B.; Jing, H.; Zhao, S.; Chen, L. Pterostilbene protects vascular endothelial cells against oxidized low-density lipoproteininduced apoptosis in vitro and in vivo. Apoptosis 2012, 17, 25-36.

(9) Satheesh, M. A.; Pari, L. The antioxidant role of pterostilbene in streptozotocinnicotinamide-induced type 2 diabetes mellitus in Wistar rats. J. Pharm. Pharmacol. 2006, 58, 1483-1490.

(10) Riche, D. M.; Riche, K. D.; Blackshear, C. T.; McEwen, C. L.; Sherman, J. J.; Wofford, M. R.; Griswold, M. E. Pterostilbene on metabolic parameters: a randomized, double-blind, and placebocontrolled trial. Evidence-Based Complementary and Alternative Medicine. 2014, 2014, 1-8.

(11) Mikstacka, R.; Rimando, A. M.; Ignatowicz, E. Antioxidant effect of trans-resveratrol, pterostilbene, quercetin and theircombinations in human erythrocytes in vitro. Plant Foods Hum. Nutr. 2010, 65 (1), 57-63.

(12) Youdim, K. A.; Shukitt-Hale, B.; MacKinnon, S.; Kalt, W.; Joseph, J. A. Polyphenolics enhance red blood cell resistance to oxidative stress: in vitro and in vivo. Biochim. Biophys. Acta, Gen. Subj. 2000, 1523, 117-122.

(13) Schmidt, L.; Baskaran, S.; Johansson, P.; Padhan, N.; Matuszewski, D.; Green, L. C.; Elfineh, L.; Wee, S.; Häggblad, M.; Martens, U.; Westermark, B.; Forsberg-Nilsson, K.; Uhrbom, L.; Claesson-Welsh, L.; Andäng, M.; Sintorn, I.; Lundgren, B.; Lönnstedt, I.; Krona, C.; Nelander, S. Case-specific potentiation of glioblastoma drugs by pterostilbene. Oncotarget. 2016, 7 (45), 73200-73215.

(14) Bofill, L.; de Sande, D.; Barbas, R.; Frontera, A.; Prohens, R. A late appearing polymorph of nutraceutical pterostilbene. CrystEng-Comm 2020, 22, 4680-4684.

(15) Bofill, L.; de Sande, D.; Barbas, R.; Prohens, R. Hydrogen Bond Polarization Overcomes Unfavorable Packing in the Most Stable High Z' Polymorph of Pterostilbene. Cryst. Growth Des. 2019, 19, 2552-2556.

(16) Liu, Y.; You, Y.; Lu, J.; Chen, X.; Yang, Z. Recent Advances in Synthesis, Bioactivity, and Pharmacokinetics of Pterostilbene, an Important Analog of Resveratrol. Molecules. 2020, 25 (5166), 1-20. 
(17) Chakraborty, A.; Gupta, N.; Ghosh, K.; Roy, P. In vitro evaluation of the cytotoxic, anti-proliferative and anti-oxidant properties of pterostilbene isolated from Pterocarpus marsupium. Toxicol. In Vitro 2010, 24, 1215-1228.

(18) Lin, W.; Valorie Leland, J. V.; Ho, C.; Pan, M. Occurrence, Bioavailability, Antiinflammatory, and Anticancer Effects of Pterostilbene. J. Agric. Food Chem. 2020, 68, 12788-12799.

(19) McCormack, D.; McFadden, D. Pterostilbene and Cancer: Current Review. J. Surg. Res. 2012, 173, 53-61.

(20) Dellinger, R. W.; Santos, S. R.; Morris, M.; Evans, M.; Alminana, D.; Guarente, L.; Marcotulli, E. Repeat dose NRPT (nicotinamide riboside and pterostilbene) increases NAD+ levels in humans safely and sustainably: a randomized, double-blind, placebocontrolled study. NPJ. Aging and Mechanisms of Disease. 2017, 3, 1-9.

(21) Smith, A. J.; Kavuru, P.; Wojtas, L.; Zaworotko, M. J.; Shytle, D. Cocrystals of Quercetin with Improved Solubility and Oral Bioavailability. Mol. Pharmaceutics 2011, 8, 1867-1876.

(22) Smith, A. J.; Kavuru, P.; Arora, K. K.; Kesani, S.; Tan, J.; Zaworotko, M. J.; Shytle, D. Crystal Engineering of Green Tea Epigallocatechin-3-gallate (EGCg) Cocrystals and Pharmacokinetic Modulation in Rats. Mol. Pharmaceutics 2013, 10, 2948-2961.

(23) Sinha, A. S.; Maguire, A. R.; Lawrence, S. E. Cocrystallization of Nutraceuticals. Cryst. Growth Des. 2015, 15, 984-1009. (24) Bofill, L.; Prohens, R.; Barbas, R.; Frontera, A. DFT analysis of uncommon $\pi \cdots \mathrm{H}$-bond arrays in a new Pterostilbene/Theophylline cocrystal. Cryst. Growth Des. 2020, 20, 6691-6698.

(25) Azzolini, M.; La Spina, M.; Mattarei, A.; Paradisi, C.; Zoratti, M.; Biasutto, L. Pharmacokinetics and tissue distribution of pterostilbene in the rat. Mol. Nutr. Food Res. 2014, 58 (11), 2122-2132.

(26) Vasisht, K.; Chadha, K.; Karan, M.; Bhalla, Y.; Kumar Jena, A.; Chadha, R. Enhancing biopharmaceutical parameters of bioflavonoid quercetin by cocrystallization. CrystEngComm $2016,18,1403-1415$.

(27) Bethune, S. J.; Schultheiss, N.; Henck, J. O. Improving the Poor Aqueous Solubility of Nutraceutical Compound Pterostilbene through Cocrystal Formation. Cryst. Growth Des. 2011, $11(7), 2817-2823$.

(28) Schultheiss, N. C. Pterostilbene cocrystals. US Patent 2011/0189277A1, 2011.

(29) Spartan'10, v 1.1.0.; Wavefunction, Inc.: Irvine, CA, 2010.

(30) SADABS; Bruker AXS: Madison, WI, USA, 2004. SAINT, Software Users Guide, Ver. 6.0; Bruker AXS: Madison, WI, USA, 1999 SADABS v2.03; Area-Detector Absorption Correction; University of Gottingen: Gottingen, Germany, 1999. Saint, Ver. 7.60A; Bruker AXS: Madison, WI, USA, 2008. SADABS, V. 2008-1; Bruker AXS: Madison, WI, USA, 2008.

(31) Sheldrick, G. M. A short history of SHELX. Acta Crystallogr., Sect. A: Found. Crystallogr. 2008, 64, 112-122.

(32) Boultif, A.; Louër, D. Indexing of powder diffraction patterns for low-symmetry lattices by the successive dichotomy method. J. Appl. Crystallogr. 1991, 24, 987-993.

(33) Gravestock, T.; Box, K.; Comer, J.; Frake, E.; Judge, S.; Ruiz, R. The "GI dissolution" method: a low volume, in vitro apparatus for assessing the dissolution/precipitation behaviour of an active pharmaceutical ingredient under biorelevant conditions. Anal. Methods 2011, 3, 560-567.

(34) Estarellas, C.; Frontera, A.; Quiñonero, D.; Deyà, P. M. Theoretical Study on Cooperativity Effects between Anion- $\pi$ and Halogen-Bonding Interactions. ChemPhysChem 2011, 12, 2742-2750.

(35) Grant, R. S.; Coggan, S. E.; Smythe, G. A. The Physiological Action of Picolinic Acid in the Human Brain. Int. J. Tryptophan Res. 2009, 2, IJTR.S2469.

(36) Cai, S.; Sato, K.; Shimizu, T.; Yamabe, S.; Hiraki, M.; Sano, C.; Tomioka, H. Antimicrobial activity of picolinic acid against extracellular and intracellular Mycobacterium 
avium complex and its combined activity with clarithromycin, rifampicin and fluoroquinolones. J. Antimicrob. Chemother. 2006, 57 (1), 85-93.

(37) Jain, R. Pterostilbene caffeine Co-Crystal: Bioavailable caffeine alternative enriched with pterostilbene. Matrix Sci. Med. 2020, 4 (1), 24-26.

(38) Yeo, S. C. M.; Ho, P. C.; Lin, H. S. Pharmacokinetics of pterostilbene in SpragueDawley rats: the impacts of aqueous solubility, fasting, dose escalation and dosing route on bioavailability. Mol. Nutr. Food Res. 2013, 57 (6), 1015-1025.

(39) Kapetanovic, I. M.; Muzzio, M.; Huang, Z.; Thompson, T. N.; McCormick, D. L. Pharmacokinetics, oral bioavailability and metabolic profile of resveratrol and its dimethylether analog, pterostilbene, in rats. Cancer Chemother. Pharmacol. 2011, 68 (3),

593-601. 
Table 1. Crystal Data of the Different Cocrystal Forms of Pterostilbene

\begin{tabular}{|c|c|c|c|c|c|}
\hline & \multicolumn{5}{|c|}{ coformer } \\
\hline & athylenedianine & DABCO & TCTD & 1,4DMP & 2,3.5-TMP \\
\hline enpirical formula & $\mathrm{C}_{17} \mathrm{H}_{20} \mathrm{NO}_{3}$ & $\mathrm{C}_{30} \mathrm{H}_{4} \mathrm{~N}_{2} \mathrm{O}_{6}$ & $\mathrm{C}_{21} \mathrm{H}_{212} \mathrm{~N}_{2} \mathrm{O}_{3}$ & $\mathrm{C}_{19} \mathrm{H}_{29} \mathrm{NO}_{3}$ & $\mathrm{C}_{90} \mathrm{H}_{42} \mathrm{~N}_{2} \mathrm{O}_{6}$ \\
\hline formula wt & 28634 & 61475 & 356.45 & 313.38 & 634.74 \\
\hline temp (K) & $100(2)$ & $100(2)$ & $100(2)$ & $100(2)$ & $100(2)$ \\
\hline ayst syst & monodinic & monoclinic & monoclinic & monodinic & tridinic \\
\hline space group & $P 2_{1} / c$ & $P_{2}{ }_{1} / c$ & $P 2_{1} / n$ & $P 2_{1} / c$ & $P 2_{1} / n$ \\
\hline$a(\mathrm{~A})$ & $11 \_3119(3)$ & $27.1201(16)$ & $8.4888(3)$ & $10.4766(4)$ & $7.9098(9)$ \\
\hline$b(\mathrm{~A})$ & $5.3890(10)$ & $6.1642(4)$ & $11.2401(5)$ & $6.6402(3)$ & $9.2397(10)$ \\
\hline$c(\mathrm{~A})$ & $26.6010(8)$ & $21.6656(13)$ & $19.8309(9)$ & $25 \operatorname{sis} 2(12)$ & $23.359(3)$ \\
\hline$a(\operatorname{deg})$ & 90 & 90 & 90 & 90 & $83699(5)$ \\
\hline$\beta(\mathrm{deg})$ & $101.0990(10)$ & $112.389(2)$ & $95.955(2)$ & $93-391(2)$ & $88.189(5)$ \\
\hline$\gamma(\mathrm{deg})$ & 90 & 90 & 90 & 90 & $79.3189(5)$ \\
\hline$V\left(A^{3}\right)$ & $1517.41(7)$ & $33489(4)$ & $1881.95(14)$ & $177398(13)$ & $16666(3)$ \\
\hline$z$ & 4 & 4 & 4 & 4 & 2 \\
\hline alod density $\left(\mathrm{Mg} / \mathrm{m}^{3}\right)$ & 1253 & 1.239 & 1.258 & 1.173 & 1.265 \\
\hline find $R$ indices $(I>2 \sigma(I))$ & $\begin{array}{c}\mathrm{R} 1=0.0375, w \mathrm{R} 2= \\
0.0912\end{array}$ & $\begin{array}{l}\mathrm{R} 1=0.0779, w \mathrm{R} 2= \\
0.2067\end{array}$ & $\begin{array}{l}\mathrm{R} 1=0.0437, w \mathrm{R2}= \\
0.1049\end{array}$ & $\begin{array}{c}\mathrm{R} 1=0.0475, w \mathrm{R} 2= \\
0.1318\end{array}$ & $\begin{array}{c}\mathrm{R1}=0.0759, \text { wR2 }= \\
0.1397\end{array}$ \\
\hline $\mathrm{CCDC}$ & 2017899 & 2017992 & 2017897 & 2017996 & 2017895 \\
\hline
\end{tabular}


Table 2. Dissolution Rate of Pterostilbene Form I and Its Caffeine, Picolinic Acid, and Ethylenediamine Cocrystals

whit form

pteratibene (form I)

pterotibene:poolinic acd oncret

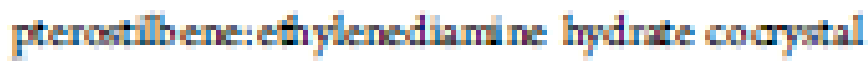

pteratibene:thylenediamine cocrytal

pterotibene:cafeine cocryal
Asolution rate (numoly min]
$1,12 \pm 2$
$427.8 \pm 30$
$2304 \pm 27$
$1761 \pm 13$
$454 \pm 9$ 
Table 3. Pharmacokinetic Parameters of Pterostilbene after Single Oral Administration of Pterostilbene form I (20 mg/kg) and Pterostilbene:Picolinic Acid Cocrystal (20 mg/kg, $13.5 \mathrm{mg}$ as Pterostilbene)a

\begin{tabular}{|c|c|c|c|c|c|c|}
\hline formulation & $C_{\max }(\mathrm{ng} / \mathrm{mL})$ & $t_{\max }(h)$ & $t_{1 / 2}(h)$ & $\mathrm{AUC}_{\mathrm{D} \sim}(\mathrm{ng} \mathrm{h} / \mathrm{mL})$ & $\operatorname{AUC}_{\text {ief }}(\mathrm{ng} \mathrm{h} / \mathrm{mL})$ & $F_{\mathrm{na}}$ \\
\hline pterostilbene & $124.8 \pm 39.3$ & $2(2-2)$ & $3.7 \pm 20$ & $575 \pm 295$ & $712 \pm 294$ & \\
\hline pterosalbene: picolinic acid cocrystal & $567.8 \pm 2842$ & $2(1-6)$ & $3.3 \pm 1.2$ & $4699 \pm 3089$ & $4772 \pm 3054$ & 9.9 \\
\hline
\end{tabular}

${ }^{a}$ Mean values \pm SD $(\mathrm{n}=5) . t_{m a}$ is the median (minimum-maximum). $F_{\text {ral }}$ is calcubted using normalized doses for pterostilbene; $F_{r i}=$

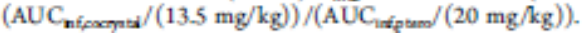




\section{Figure Captions}

Figure 1. MEPS of pterostilbene on the 0.002 bohr $\AA-3$ electron density isosurface calculated with Spartan29 at the DFT level of computation using a B3LYP/6-31G* basis set. Red and blue isosurfaces represent negative and positive values of MEP.

Figure 2.. Crystal structure of pterostilbene:ethylenediamine cocrystal. Some of the most relevant interactions have been represented.

Figure 3. Crystal structure of the pterostilbene:DABCO cocrystal. Some of the most relevant interactions have been represented.

Figure 4. Crystal structure of the pterostilbene:1,4,8,11-tetraazacyclotetradecane cocrystal. Some of the most relevant interactions have been represented.

Figure 5. Crystal structure of the pterostilbene:1,4-dimethylpiperazine cocrystal. Some of the most relevant interactions have been represented.

Figure 6. Crystal structure of the pterostilbene:2,3,5-trimethylpyrazine cocrystal. Some of the most relevant interactions have been represented.

Figure 7. Average dissolution rate profiles of pterostilbene form I (blue), pterostilbene:caffeine cocrystal (purple), pterostilbene:picolinic acid cocrystal (red), anhydrous pterostilbene:ethylenediamine cocrystal (green), and pterostilbene:ethylenediamine hydrate cocrystal (gray).

Figure 8. Pharmacokinetic profiles of pterostilbene in male SD rats following a single oral administration of pterostilbene form I $(20 \mathrm{mg} / \mathrm{kg})$ and pterostilbene:picolinic acid cocrystal (20 $\mathrm{mg} / \mathrm{kg}, 13.5 \mathrm{mg}$ as pterostilbene). Mean values $(\mathrm{n}=5)$. 
Figure 1

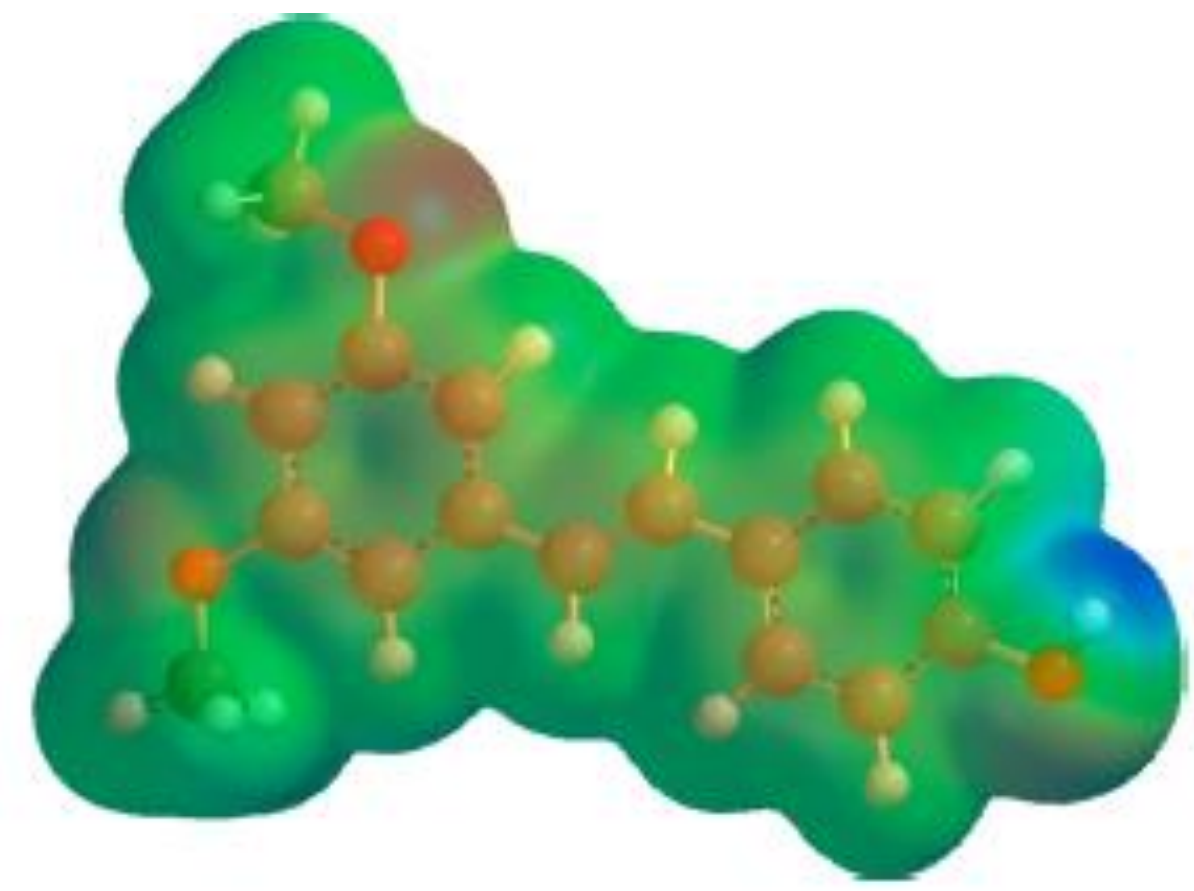


Figure 2

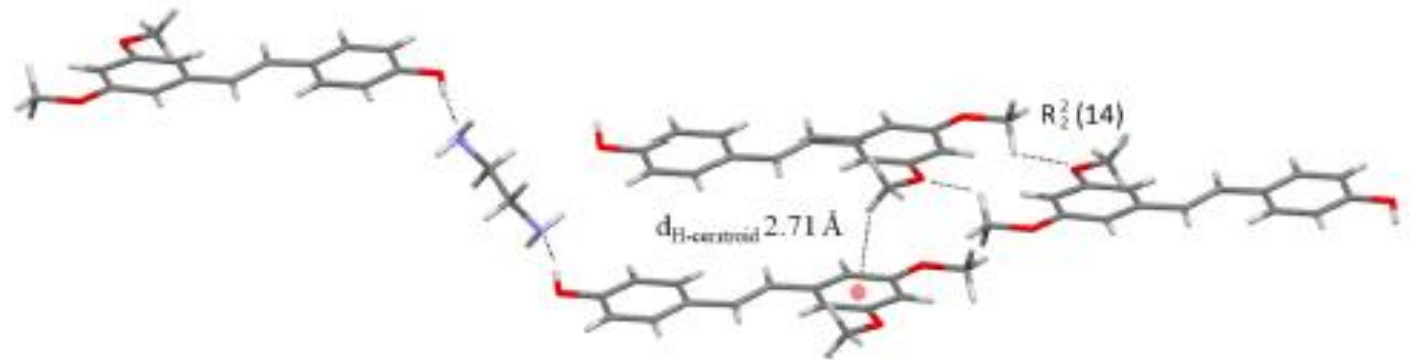


Figure 3

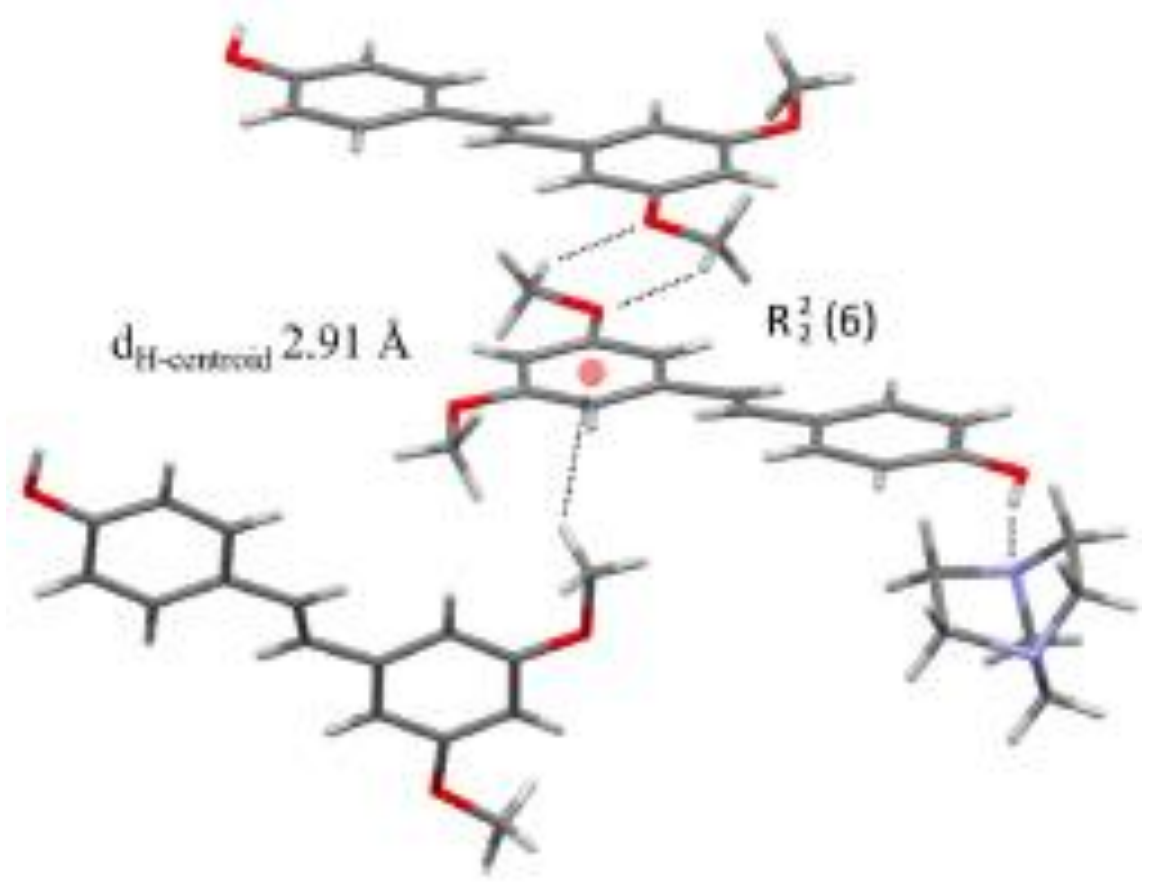


Figure 4

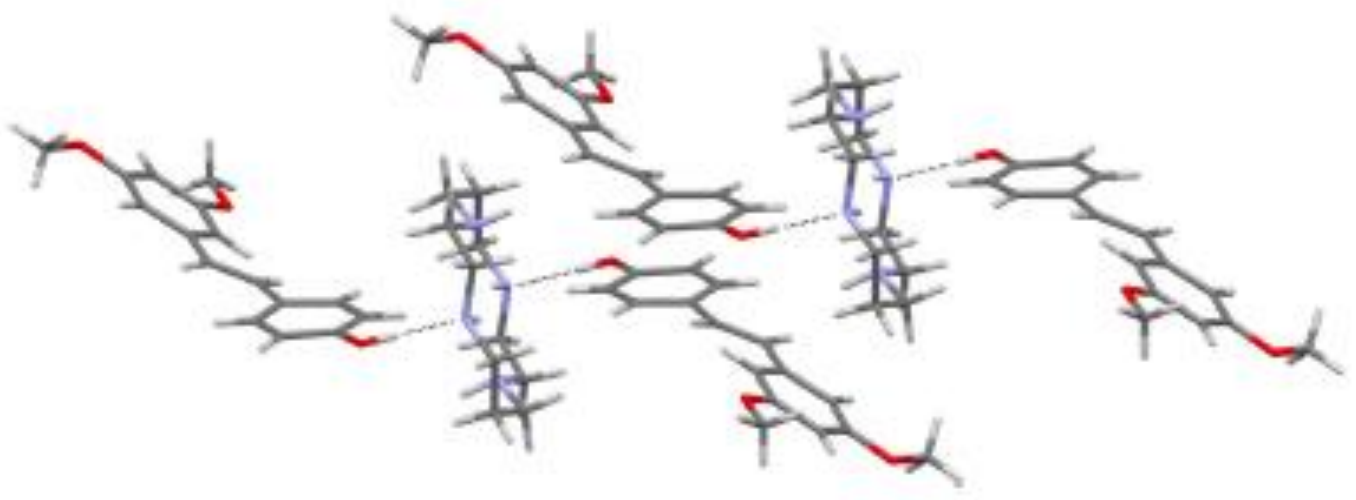


Figure 5

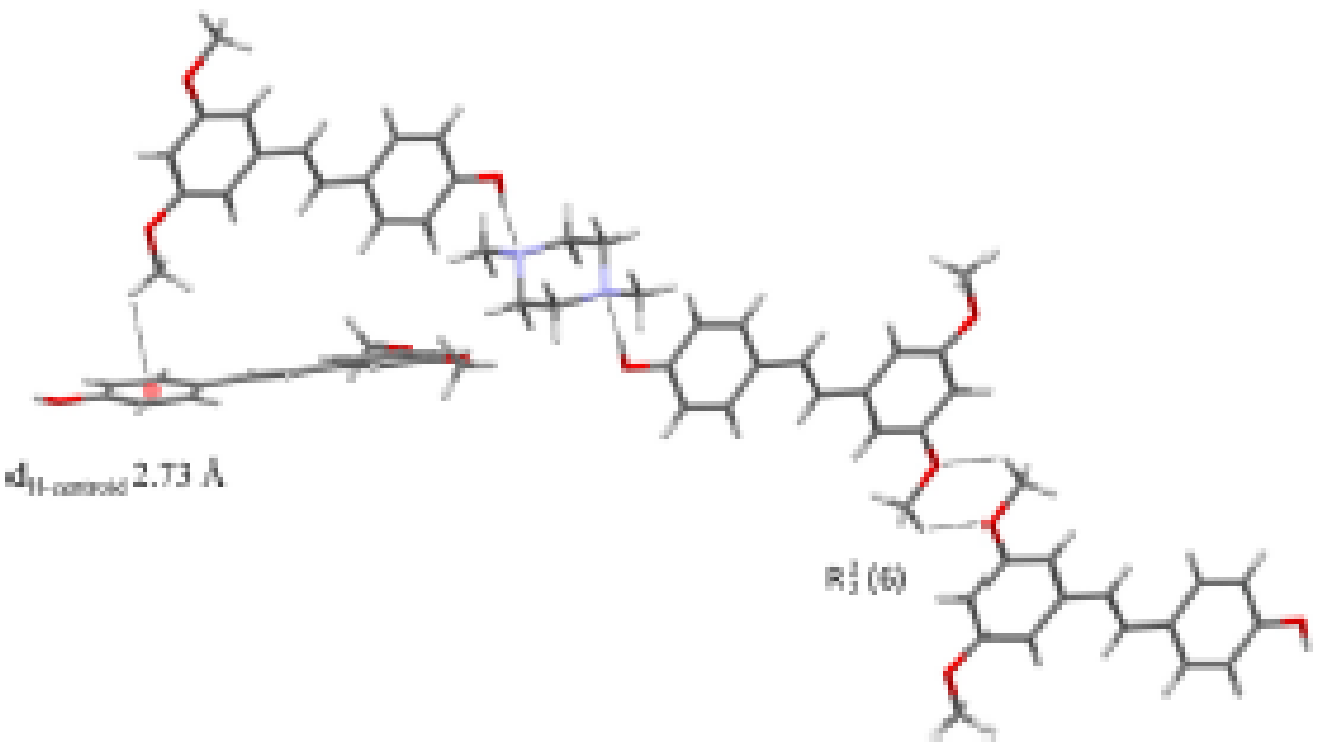


Figure 6

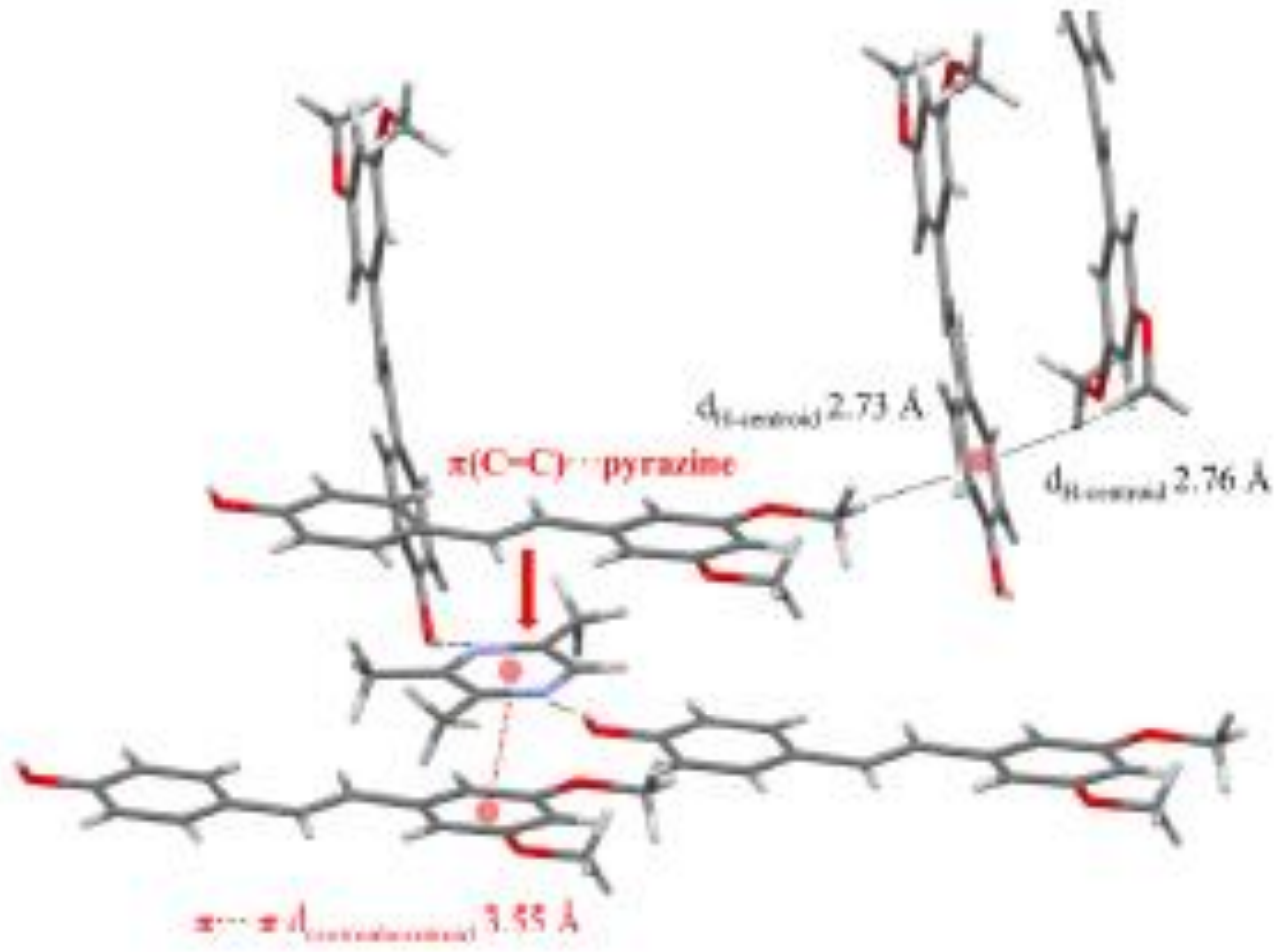


Figure 7

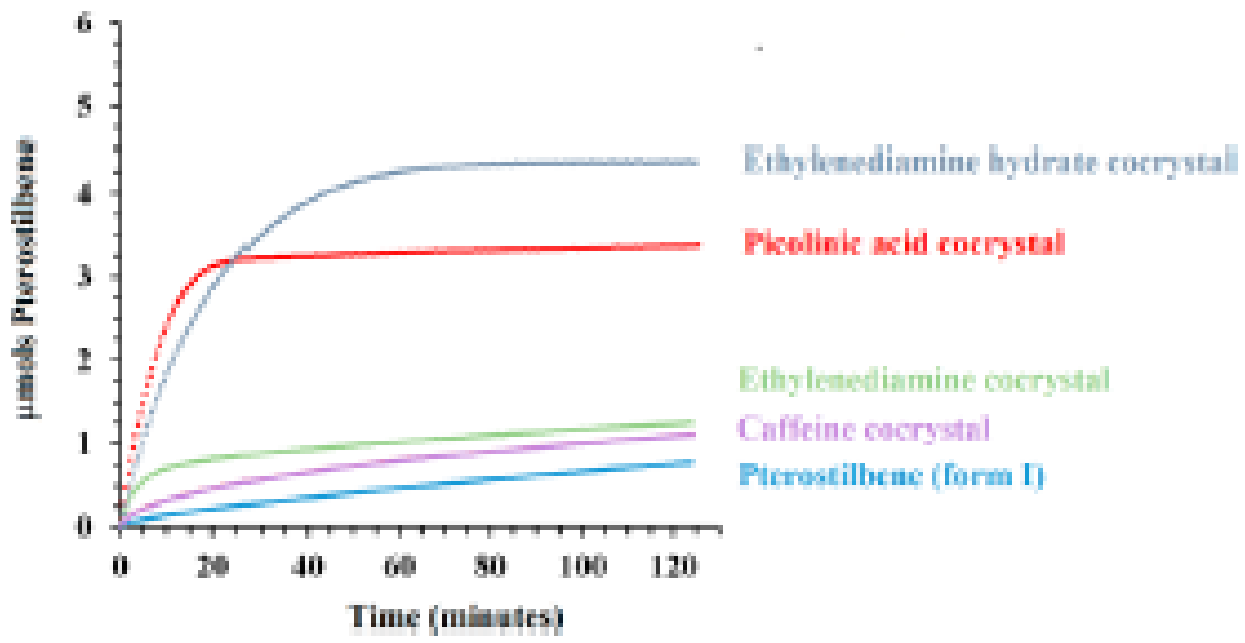


Figure 8

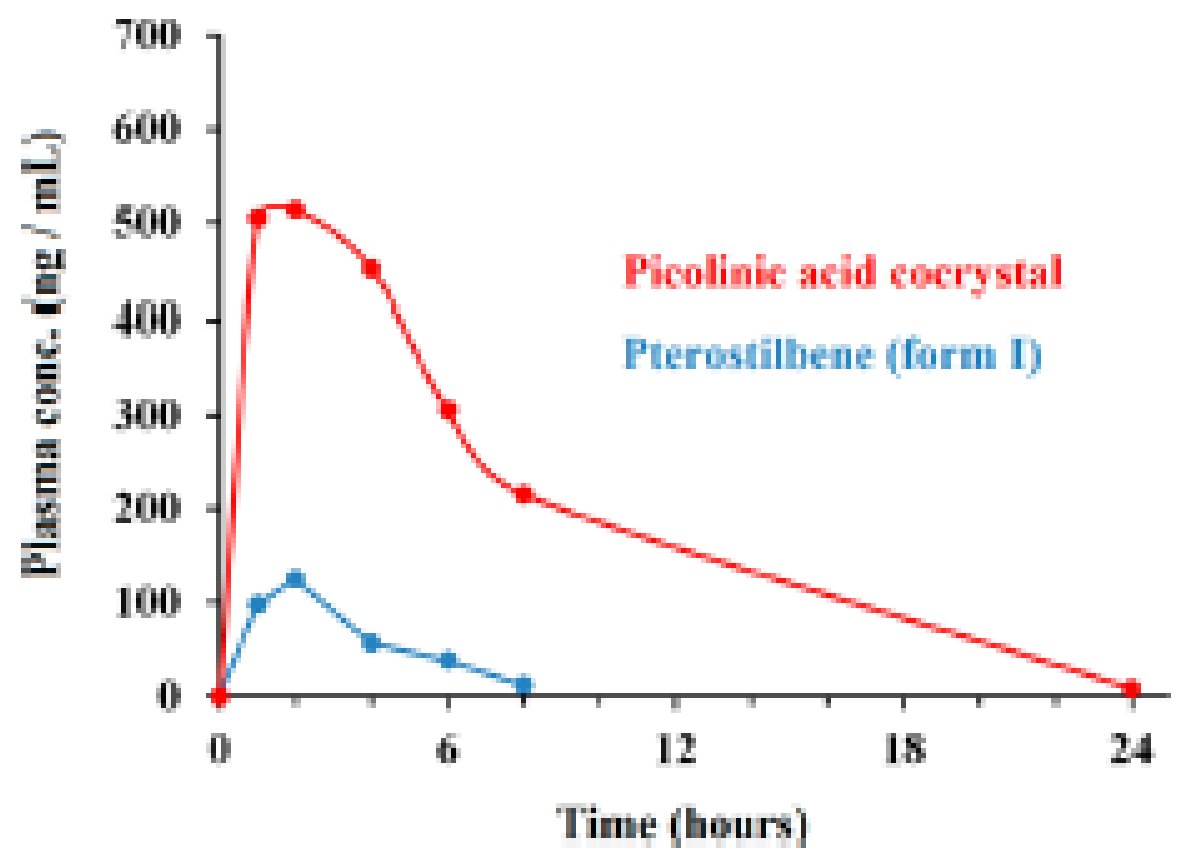

